\title{
Conformal Killing-Yano Tensors on Manifolds with Mixed 3-Structures ${ }^{\star}$
}

\author{
Stere IANUŞ ${ }^{\dagger}$, Mihai VISINESCU ${ }^{\ddagger}$ and Gabriel Eduard VÎLCU ${ }^{\dagger \S}$ \\ $\dagger$ University of Bucharest, Faculty of Mathematics and Computer Science, \\ Str. Academiei, Nr. 14, Sector 1, Bucharest 70109, Romania \\ E-mail: ianus@gta.math.unibuc.ro \\ $\ddagger$ National Institute for Physics and Nuclear Engineering, Department of Theoretical Physics, \\ P.O. Box M.G.-6, Magurele, Bucharest, Romania \\ E-mail: mvisin@theory.nipne.ro \\ URL: http://www.theory.nipne.ro/ mvisin/ \\ $\S$ Petroleum-Gas University of Ploieşti, Department of Mathematics and Computer Science, \\ Bulevardul Bucureşti, Nr. 39, Ploieşti 100680, Romania \\ E-mail: gvilcu@mail.upg-ploiesti.ro
}

Received October 30, 2008, in final form February 16, 2009; Published online February 23, 2009 doi:10.3842/SIGMA.2009.022

\begin{abstract}
We show the existence of conformal Killing-Yano tensors on a manifold endowed with a mixed 3-Sasakian structure.
\end{abstract}

Key words: Killing-Yano tensor; mixed 3-structure; Einstein space

2000 Mathematics Subject Classification: 53C15; $81 \mathrm{~T} 20$

\section{Introduction}

Investigations of the properties of space-times of higher dimensions $(D>4)$ have recently attracted considerable attention as a result of their appearance in theories of unification such as string and $M$ theories.

It is important to study the symmetries of these space-times in order to characterize the geodesic motions in them. In general a space-time could possess explicit and hidden symmetries encoded in the multitude of Killing vectors and higher Killing tensors respectively. The symmetries allow one to define conserved quantities along geodesics linear and polynomial in canonical momenta. Their existence guarantees the integrability of the geodesic motions and is intimately related to separability of Hamilton-Jacobi (see, e.g. [3]) and the Klein-Gordon equation [9] at the quantum level.

The next most simple objects that can be studied in connection with the symmetries of a manifold after Killing tensors are Killing-Yano tensor [37]. Their physical interpretation remained obscure until Floyd [13] and Penrose [32] showed that the Killing tensor $K^{\mu \nu}$ of the 4-dimensional Kerr-Newman space-time admits a certain square-root which defines a KillingYano tensor. Subsequently it was realized [15] that a Killing-Yano tensor generate additional supercharges in the dynamics of pseudo-classical spinning particles. In this way it was realized the natural connection between Killing-Yano tensors and supersymmetries [33]. Passing to

${ }^{\star}$ This paper is a contribution to the Proceedings of the XVIIth International Colloquium on Integrable Systems and Quantum Symmetries (June 19-22, 2008, Prague, Czech Republic). The full collection is available at http://www.emis.de/journals/SIGMA/ISQS2008.html 
quantum Dirac equation it was discovered [10] that Killing-Yano tensors generate conserved non-standard Dirac operators which commute with the standard one.

The conformal extension of the Killing tensor equation determines the conformal Killing tensors [23] which define first integrals of the null geodesic equation. Investigation of the properties of higher-dimensional space-times has pointed out the role of the conformal Killing-Yano tensors to generate background metrics with black-hole solutions (see, e.g. [14]).

The aim of this paper is to investigate the existence of conformal Killing-Yano tensors on some manifolds endowed with special structures which could be relevant in the theories of modern physics $[8,15,26,35,36]$.

Versions of $M$-theory could be formulated in a space-time with various number of time dimensions giving rise to exotic space-time signatures. The $M$-theory in $10+1$ dimensions is linked via dualities to a $M^{*}$ theory in $9+2$ dimensions and a $M^{\prime}$ theory in $6+5$ dimensions. Various limits of these will give rise to $I I A$ - and $I I B$-like string theories in many variants of dimensions and signatures [18].

The paraquaternionic structures arise in a natural way in theoretical physics, both in string theory and integrable systems $[11,12,17,31]$. The counterpart in odd dimension of a paraquaternionic structure was introduced in [19]. It is called mixed 3-structure, which appears in a natural way on lightlike hypersurfaces in paraquaternionic manifolds. A compatible metric with a mixed 3 -structures is necessarily semi-Riemann and mixed 3-Sasakian manifolds are Einstein [7, 20], hence the possible importance of these structures in theoretical physics.

\section{Killing vector fields and their generalizations}

Let $(M, g)$ be a semi-Riemannian manifold. A vector field $X$ on $M$ is said to be a Killing vector field if the Lie derivative with respect to $X$ of the metric $g$ vanishes:

$$
L_{X} g=0 .
$$

Alternatively, if $\nabla$ denotes the Levi-Civita connection of $g$, then (1) can be rewritten as

$$
g\left(\nabla_{Y} X, Z\right)+g\left(Y, \nabla_{Z} X\right)=0,
$$

for all vector fields $Y, Z$ on $M$.

It is easy to see that the Lie bracket of two Killing fields is still a Killing field and thus the Killing fields on a manifold $M$ form a Lie subalgebra of vector fields on $M$. This is the Lie algebra of the isometry group of the manifold $M$, provided that $M$ is compact.

Killing vector fields can be generalized to conformal Killing vector fields [37], i.e. vector fields with a flow preserving a given conformal class of metrics. A natural generalization of conformal Killing vector fields is given by the conformal Killing-Yano tensors [25]. Roughly speaking, a conformal Killing-Yano tensor of rank $p$ on a semi-Riemannian manifold $(M, g)$ is a $p$-form $\omega$ which satisfies:

$$
\left.\nabla_{X} \omega=\frac{1}{p+1} X\right\lrcorner d \omega-\frac{1}{n-p+1} X^{*} \wedge d^{*} \omega
$$

for any vector field $X$ on $M$, where $\nabla$ is the Levi-Civita connection of $g, n$ is the dimension of $M, X^{*}$ is the 1 -form dual to the vector field $X$ with respect to the metric $\left.g,\right\lrcorner$ is the operator dual to the wedge product and $d^{*}$ is the adjoint of the exterior derivative $d$. If $\omega$ is co-closed in (2), then we obtain the definition of a Killing-Yano tensor (introduced by Yano [37]). We can easily see that for $p=1$, they are dual to Killing vector fields.

We remark that Killing-Yano tensors are also called Yano tensors or Killing forms, and conformal Killing-Yano tensors are sometimes referred as conformal Yano tensors, conformal Killing forms or twistor forms [2, 29, 34]. 
For generalizations of the Killing vectors one might also consider higher order symmetric tensors. Let $\rho$ be a covariant symmetric tensor field of rank $r$ on a semi-Riemannian manifold $(M, g)$. Then $\rho$ is a Killing tensor field if the symmetrization of covariant derivative of $\rho$ vanishes identically:

$$
\nabla_{(\lambda} \rho_{\left.\mu_{1} \ldots \mu_{r}\right)}=0 .
$$

If the Ricci tensor of a semi-Riemannian manifold $(M, g)$ is Killing, then $(M, g)$ is an Einstein-like space [16].

The relevance in physics of the Killing tensors is given by the following proposition which could be easily proved:

Proposition 1. A symmetric tensor $\rho$ on $M$ is a Killing tensor if and only if the quantity

$$
K=\rho_{\mu_{1} \ldots \mu_{r}} \dot{c}^{\mu_{1}} \cdots \dot{c}^{\mu_{r}}
$$

is constant along every geodesic c in $M$.

Here the over-dot denotes the ordinary proper time derivative and the proposition ensures that $K$ is a first integral of the geodesic equation.

These two generalizations of the Killing vector equation could be related. Let $\omega_{\mu_{1} \ldots \mu_{p}}$ be a Killing-Yano tensor, then the tensor field

$$
\rho_{\mu \nu}=\omega_{\mu \mu_{2} \ldots \mu_{p}} \omega_{\nu}^{\mu_{2} \ldots \mu_{p}}
$$

is a Killing tensor and it sometimes refers to it as the associated tensor with $\omega$. However, the converse statement is not true in general: not all Killing tensors of rank 2 are associated with a Killing-Yano tensor. That is the case of the spaces which admit Killing tensors but no Killing-Yano tensors.

\section{Manifolds with mixed 3-structures}

Let $M$ be a differentiable manifold equipped with a triple $(\phi, \xi, \eta)$, where $\phi$ is a a field of endomorphisms of the tangent spaces, $\xi$ is a vector field and $\eta$ is a 1-form on $M$ such that:

$$
\phi^{2}=-\epsilon I+\eta \otimes \xi, \quad \eta(\xi)=\epsilon .
$$

If $\epsilon=1$ then $(\phi, \xi, \eta)$ is said to be an almost contact structure on $M$ (see [5]), and if $\epsilon=-1$ then $(\phi, \xi, \eta)$ is said to be an almost paracontact structure on $M$ (see [27]).

Definition 1 ([19]). Let $M$ be a differentiable manifold which admits an almost contact structure $\left(\phi_{1}, \xi_{1}, \eta_{1}\right)$ and two almost paracontact structures $\left(\phi_{2}, \xi_{2}, \eta_{2}\right)$ and $\left(\phi_{3}, \xi_{3}, \eta_{3}\right)$, satisfying the following conditions:

$$
\begin{aligned}
& \eta_{\alpha}\left(\xi_{\beta}\right)=0, \quad \forall \alpha \neq \beta \\
& \phi_{\alpha}\left(\xi_{\beta}\right)=-\phi_{\beta}\left(\xi_{\alpha}\right)=\epsilon_{\gamma} \xi_{\gamma} \\
& \eta_{\alpha} \circ \phi_{\beta}=-\eta_{\beta} \circ \phi_{\alpha}=\epsilon_{\gamma} \eta_{\gamma} \\
& \phi_{\alpha} \phi_{\beta}-\eta_{\beta} \otimes \xi_{\alpha}=-\phi_{\beta} \phi_{\alpha}+\eta_{\alpha} \otimes \xi_{\beta}=\epsilon_{\gamma} \phi_{\gamma}
\end{aligned}
$$

where $(\alpha, \beta, \gamma)$ is an even permutation of $(1,2,3)$ and $\epsilon_{1}=1, \epsilon_{2}=\epsilon_{3}=-1$.

Then the manifold $M$ is said to have a mixed 3-structure $\left(\phi_{\alpha}, \xi_{\alpha}, \eta_{\alpha}\right)_{\alpha=\overline{1,3}}$. 
Definition 2. If a manifold $M$ with a mixed 3-structure $\left(\phi_{\alpha}, \xi_{\alpha}, \eta_{\alpha}\right)_{\alpha=\overline{1,3}}$ admits a semiRiemannian metric $g$ such that:

$$
g\left(\phi_{\alpha} X, \phi_{\alpha} Y\right)=\epsilon_{\alpha} g(X, Y)-\eta_{\alpha}(X) \eta_{\alpha}(Y), \quad g\left(X, \xi_{\alpha}\right)=\eta_{\alpha}(X)
$$

for all $X, Y \in \Gamma(T M)$ and $\alpha=1,2,3$, then we say that $M$ has a metric mixed 3-structure and $g$ is called a compatible metric. Moreover, if $\left(\phi_{1}, \xi_{1}, \eta_{1}, g\right)$ is a Sasakian structure, i.e. (see [5]):

$$
\left(\nabla_{X} \phi_{1}\right) Y=g(X, Y) \xi_{1}-\eta_{1}(Y) X
$$

and $\left(\phi_{2}, \xi_{2}, \eta_{2}, g\right),\left(\phi_{3}, \xi_{3}, \eta_{3}, g\right)$ are LP-Sasakian structures, i.e. (see [27]):

$$
\begin{aligned}
& \left(\nabla_{X} \phi_{2}\right) Y=g\left(\phi_{2} X, \phi_{2} Y\right) \xi_{2}+\eta_{2}(Y) \phi_{2}^{2} X, \\
& \left(\nabla_{X} \phi_{3}\right) Y=g\left(\phi_{3} X, \phi_{3} Y\right) \xi_{3}+\eta_{3}(Y) \phi_{3}^{2} X,
\end{aligned}
$$

then $\left(\left(\phi_{\alpha}, \xi_{\alpha}, \eta_{\alpha}\right)_{\alpha=\overline{1,3}}, g\right)$ is said to be a mixed Sasakian 3-structure on $M$.

We remark that if $\left(M,\left(\phi_{\alpha}, \xi_{\alpha}, \eta_{\alpha}\right)_{\alpha=\overline{1,3}}, g\right)$ is a manifold with a metric mixed 3-structure then the signature of $g$ is $(2 n+1,2 n+2)$ and the dimension of the manifold $M$ is $4 n+3$ because one can check that, at each point of $M$, always there exists a pseudo-orthonormal frame field given by $\left\{\left(E_{i}, \phi_{1} E_{i}, \phi_{2} E_{i}, \phi_{3} E_{i}\right)_{i=\overline{1, n}}, \xi_{1}, \xi_{2}, \xi_{3}\right\}$.

The main property of a manifold endowed with a mixed 3-Sasakian structure is given by the following theorem (see $[7,20])$ :

Theorem 1. Any $(4 n+3)$-dimensional manifold endowed with a mixed 3-Sasakian structure is an Einstein space with Einstein constant $\lambda=4 n+2$.

Concerning the symmetric Killing tensors let us note that D.E. Blair studied in [4] the almost contact manifold with Killing structure tensors. He assumed that $M$ has an almost contact metric structure $(\phi, \xi, \eta, g)$ such that $\phi$ and $\eta$ are Killing. Then he proved that if $(\phi, \xi, \eta, g)$ is normal, i.e. the Nijenhuis tensor $N_{\phi}$ satisfies (see [5]):

$$
N_{\phi}+2 d \eta \otimes \xi=0,
$$

then $(\phi, \xi, \eta, g)$ is a cosymplectic structure, i.e. $\Omega$ and $\eta$ are closed, where $\Omega$ is the fundamental 2-form of the manifold defined by

$$
\Omega(X, Y)=g(\phi X, Y),
$$

for all vector fields $X, Y$ on $M$.

For a mixed 3-structure with a compatible semi-Riemannian metric $g$, we have the following result.

Proposition 2. Let $(M, g)$ be a semi-Riemannian manifold. If $(M, g)$ has a mixed 3-Sasakian structure $\left(\phi_{\alpha}, \xi_{\alpha}, \eta_{\alpha}\right)_{\alpha=\overline{1,3}}$, then $\left(\phi_{\alpha}\right)_{\alpha=\overline{1,3}}$ cannot be Killing-Yano tensor fields.

Proof. If $\left(\phi_{1}, \xi_{1}, \eta_{1}, g\right)$ is a Sasakian structure, from (4) we obtain:

$$
\left(\nabla_{X} \phi_{1}\right) X=g(X, X) \xi_{1} \neq 0
$$

for any non-lightlike vector field $X$ orthogonal to $\xi_{1}$.

For LP-Sasakian structures $\left(\phi_{2}, \xi_{2}, \eta_{2}, g\right)$ and $\left(\phi_{3}, \xi_{3}, \eta_{3}, g\right)$, from (5) and (6) we have:

$$
\left(\nabla_{X} \phi_{\alpha}\right) X=g\left(\phi_{\alpha} X, \phi_{\alpha} X\right) \xi_{\alpha} \neq 0, \quad \alpha \in\{2,3\}
$$

for any non-lightlike vector field $X$ orthogonal to $\xi_{2}$. 
Theorem 2. Let $(M, g)$ be a semi-Riemannian manifold. If $(M, g)$ admits a mixed 3-Sasakian structure, then any conformal Killing vector field on $(M, g)$ is a Killing vector field.

Proof. A vector field $X$ on $M$ is conformal Killing iff

$$
L_{X} g=f \cdot g,
$$

for $f \in C^{\infty}(M, \mathbb{R})$.

From (7) we have

$$
\left(L_{X} g\right)\left(\xi_{\alpha}, \xi_{\alpha}\right)=f g\left(\xi_{\alpha}, \xi_{\alpha}\right)=\epsilon_{\alpha} f .
$$

But, by the Lie operator's properties,

$$
\begin{aligned}
\left(L_{X} g\right)\left(\xi_{\alpha}, \xi_{\alpha}\right) & =X g\left(\xi_{\alpha}, \xi_{\alpha}\right)-2 g\left(L_{X} \xi_{\alpha}, \xi_{\alpha}\right)=-2 g\left(\left[X, \xi_{\alpha}\right], \xi_{\alpha}\right) \\
& =-2 g\left(\nabla_{X} \xi_{\alpha}, \xi_{\alpha}\right)+2 g\left(\nabla_{\xi_{\alpha}} X, \xi_{\alpha}\right)=2 \epsilon_{\alpha} g\left(\phi_{\alpha} X, \xi_{\alpha}\right)-2 g\left(X, \nabla_{\xi_{\alpha}} \xi_{\alpha}\right)=0
\end{aligned}
$$

because $\phi_{\alpha} X \perp \xi_{\alpha}(\alpha \in\{1,2,3\})$ and $\nabla_{\xi_{\alpha}} \xi_{\alpha}=0$.

Consequently,

$$
f=\epsilon_{\alpha}\left(L_{X} g\right)\left(\xi_{\alpha}, \xi_{\alpha}\right)=0,
$$

so that $L_{X} g=0$, i.e. $X$ is Killing vector field.

Actually this result holds on Sasakian, LP-Sasakian and 3-Sasakian manifolds with the same proof.

\section{Killing vector fields on manifolds with mixed 3-structures}

An almost para-hypercomplex structure on a smooth manifold $M$ is a triple $H=\left(J_{\alpha}\right)_{\alpha=\overline{1,3}}$, where $J_{1}$ is an almost complex structure on $M$ and $J_{2}, J_{3}$ are almost product structures on $M$, satisfying: $J_{1} J_{2} J_{3}=-\mathrm{Id}$. In this case $(M, H)$ is said to be an almost para-hypercomplex manifold.

A semi-Riemannian metric $g$ on $(M, H)$ is said to be para-hyperhermitian if it satisfies:

$$
g\left(J_{\alpha} X, J_{\alpha} Y\right)=\epsilon_{\alpha} g(X, Y), \quad \alpha \in\{1,2,3\},
$$

for all $X, Y \in \Gamma(T M)$, where $\epsilon_{1}=1, \epsilon_{2}=\epsilon_{3}=-1$. In this case, $(M, g, H)$ is called an almost para-hyperhermitian manifold. Moreover, if each $J_{\alpha}$ is parallel with respect to the Levi-Civita connection of $g$, then $(M, g, H)$ is said to be a para-hyper-Kähler manifold.

Theorem 3. Let $(M, g)$ be a semi-Riemannian manifold. Then the following five assertions are mutually equivalent:

(i) $(M, g)$ admits a mixed 3-Sasakian structure.

(ii) The cone $(C(M), \bar{g})=\left(M \times \mathbb{R}_{+}, d r^{2}+r^{2} g\right)$ admits a para-hyper-Kähler structure.

(iii) There exists three orthogonal Killing vector fields $\left\{\xi_{1}, \xi_{2}, \xi_{3}\right\}$ on $M$, with $\xi_{1}$ unit spacelike vector field and $\xi_{2}, \xi_{3}$ unit timelike vector fields satisfying

$$
\left[\xi_{\alpha}, \xi_{\beta}\right]=\left(\epsilon_{\alpha}+\epsilon_{\beta}\right) \epsilon_{\gamma} \xi_{\gamma}
$$

where $(\alpha, \beta, \gamma)$ is an even permutation of $(1,2,3)$ and $\epsilon_{1}=1, \epsilon_{2}=\epsilon_{3}=-1$, such that the tensor fields $\phi_{\alpha}$ of type $(1,1)$, defined by:

$$
\phi_{\alpha} X=-\epsilon_{\alpha} \nabla_{X} \xi_{\alpha}, \quad \alpha \in\{1,2,3\},
$$

satisfies the conditions (4), (5) and (6). 
(iv) There exists three orthogonal Killing vector fields $\left\{\xi_{1}, \xi_{2}, \xi_{3}\right\}$ on $M$, with $\xi_{1}$ unit spacelike vector field and $\xi_{2}, \xi_{3}$ unit timelike vector fields satisfying (9), such that:

$$
R\left(X, \xi_{\alpha}\right) Y=g\left(\xi_{\alpha}, Y\right) X-g(X, Y) \xi_{\alpha}, \quad \alpha \in\{1,2,3\}
$$

where $R$ is the Riemannian curvature tensor of the Levi-Civita connection $\nabla$ of $g$.

$(v)$ There exists three orthogonal Killing vector fields $\left\{\xi_{1}, \xi_{2}, \xi_{3}\right\}$ on $M$, with $\xi_{1}$ unit spacelike vector field and $\xi_{2}, \xi_{3}$ unit timelike vector fields satisfying (9), such that the sectional curvature of every section containing $\xi_{1}, \xi_{2}$ or $\xi_{3}$ equals 1.

Proof. $(i) \Rightarrow(i i)$. If $\left(M^{4 n+3},\left(\phi_{\alpha}, \xi_{\alpha}, \eta_{\alpha}\right)_{\alpha=\overline{1,3}}, g\right)$ is a manifold endowed with a mixed 3Sasakian structure, then we can define a para-hyper-Kähler structure $\left\{J_{\alpha}\right\}_{\alpha=\overline{1,3}}$ on the cone $(C(M), \bar{g})=\left(M \times \mathbb{R}_{+}, d r^{2}+r^{2} g\right)$, by:

$$
J_{\alpha} X=\phi_{\alpha} X-\eta_{\alpha}(X) \Phi, \quad J_{\alpha} \Phi=\xi_{\alpha},
$$

for any $X \in \Gamma(T M)$ and $\alpha \in\{1,2,3\}$, where $\Phi=r \partial_{r}$ is the Euler field on $C(M)$ (see also [6]).

$($ ii $) \Rightarrow(i)$. If the cone $(C(M), \bar{g})=\left(M \times \mathbb{R}_{+}, d r^{2}+r^{2} g\right)$ admits a para-hyper-Kähler structure $\left\{J_{\alpha}\right\}_{\alpha=\overline{1,3}}$, then we can identify $M$ with $M \times\{1\}$ and we have a mixed 3-Sasakian structure $\left(\left(\phi_{\alpha}, \xi_{\alpha}, \eta_{\alpha}\right)_{\alpha=\overline{1,3}}, g\right)$ on $M$ given by:

$$
\xi_{\alpha}=J_{\alpha}\left(\partial_{r}\right), \quad \phi_{\alpha} X=-\epsilon_{\alpha} \nabla_{X} \xi_{\alpha}, \quad \eta_{\alpha}(X)=g\left(\xi_{\alpha}, X\right)
$$

for any $X \in \Gamma(T M)$ and $\alpha \in\{1,2,3\}$.

$($ ii $) \Rightarrow($ iii $)$. If the cone $C(M)$ admits a para-hyper-Kähler structure $\left\{J_{\alpha}\right\}_{\alpha=\overline{1,3}}$, then using the above identifications, we can view all vector fields $X, Y$ on $M$ as vector fields on the $C(M)$. If $\bar{\nabla}$ is the Levi-Civita connection of $\bar{g}$, then we have the following formulas (see [30]):

$$
\bar{\nabla}_{X} Y=\nabla_{X} Y-r g(X, Y) \partial_{r}, \quad \bar{\nabla}_{\partial_{r}} X=\bar{\nabla}_{X} \partial_{r}=\frac{1}{r} X, \quad \bar{\nabla}_{\partial_{r}} \partial_{r}=0
$$

On the other hand, because $(i i) \Rightarrow(i)$, we have that $\left(\left(\phi_{\alpha}, \xi_{\alpha}, \eta_{\alpha}\right)_{\alpha=\overline{1,3}}, g\right)$ given by (11) is a mixed 3-Sasakian structure on $M$ and the conditions (4), (5) and (6) are satisfied.

Then, using (8), (11) and (12), since $J_{1}, J_{2}$ and $J_{3}$ are parallel, we obtain for all vector fields $X, Y$ on $M$ and $\alpha \in\{1,2,3\}$ :

$$
\begin{aligned}
& g\left(\nabla_{X} \xi_{\alpha}, Y\right)+g\left(X, \nabla_{Y} \xi_{\alpha}\right)=\bar{g}\left(\bar{\nabla}_{X} \xi_{\alpha}+r g\left(X, \xi_{\alpha}\right) \partial_{r}, Y\right)+\bar{g}\left(X, \bar{\nabla}_{Y} \xi_{\alpha}+r g\left(Y, \xi_{\alpha}\right) \partial_{r}\right) \\
& \quad=\bar{g}\left(\bar{\nabla}_{X}\left(J_{\alpha} \partial_{r}\right), Y\right)+\bar{g}\left(X, \bar{\nabla}_{Y}\left(J_{\alpha} \partial_{r}\right)\right)=\bar{g}\left(J_{\alpha} \bar{\nabla}_{X} \partial_{r}, Y\right)+\bar{g}\left(X, J_{\alpha} \bar{\nabla}_{Y} \partial_{r}\right) \\
& \quad=\frac{1}{r}\left(\bar{g}\left(J_{\alpha} X, Y\right)+\bar{g}\left(X, J_{\alpha} Y\right)\right)=0
\end{aligned}
$$

and consequently $\xi_{\alpha}$ is a Killing vector field, for $\alpha=1,2,3$.

From (11) we also obtain:

$$
g\left(\xi_{\alpha}, \xi_{\alpha}\right)=\eta_{\alpha}\left(\xi_{\alpha}\right)=\epsilon_{\alpha}, \quad \alpha \in\{1,2,3\}
$$

and

$$
g\left(\xi_{\alpha}, \xi_{\beta}\right)=\eta_{\beta}\left(\xi_{\alpha}\right)=0, \quad \forall \alpha \neq \beta
$$

Consequently, $\left\{\xi_{1}, \xi_{2}, \xi_{3}\right\}$ are orthogonal Killing vector fields on $M$, with $\xi_{1}$ unit spacelike vector field and $\xi_{2}, \xi_{3}$ unit timelike vector fields. 
Moreover we have:

$$
\left[\xi_{\alpha}, \xi_{\beta}\right]=\nabla_{\xi_{\alpha}} \xi_{\beta}-\nabla_{\xi_{\beta}} \xi_{\alpha}=-\epsilon_{\beta} \phi_{\beta} \xi_{\alpha}+\epsilon_{\alpha} \phi_{\alpha} \xi_{\beta}=\left(\epsilon_{\alpha}+\epsilon_{\beta}\right) \epsilon_{\gamma} \xi_{\gamma}
$$

for any even permutation $(\alpha, \beta, \gamma)$ of $(1,2,3)$.

$($ iii $) \Rightarrow($ ii $)$. If $\left\{\xi_{1}, \xi_{2}, \xi_{3}\right\}$ are orthogonal Killing vector fields on $M$, such that $\xi_{1}$ is unit spacelike, $\xi_{2}, \xi_{3}$ are unit timelike vector fields and relations (4), (5) and (6) are satisfied, then we can define a para-hyper-Kähler structure on the cone $(C(M), \bar{g})=\left(M \times \mathbb{R}_{+}, d r^{2}+r^{2} g\right)$, by (10), where $\eta_{\alpha}$ is the 1 -form dual to $\xi_{\alpha}$, for $\alpha \in\{1,2,3\}$.

$($ iii $) \Leftrightarrow(i v)$. This equivalence follows from straightforward computations using the expression of the Riemannian curvature tensor $R$.

$(i v) \Leftrightarrow(v)$. This equivalence is a simple calculation, using the formula of the sectional curvature:

$$
K(\pi)=\frac{R(X, Y, X, Y)}{g(X, X) g(Y, Y)-g(X, Y)^{2}},
$$

for a non-degenerate 2-plane $\pi$ spanned by $\{X, Y\}$.

Corollary 1. Let $\left(M^{4 n+3},\left(\phi_{\alpha}, \xi_{\alpha}, \eta_{\alpha}\right)_{\alpha=\overline{1,3}}, g\right)$ be a manifold endowed with a mixed 3-Sasakian structure. Then:

(i) $\xi_{1}$ is unit spacelike Killing vector field and $\xi_{2}$, $\xi_{3}$ are unit timelike Killing vector fields on $M$.

(ii) $\eta_{1}, \eta_{2}, \eta_{3}$ are conformal Killing-Yano tensors of rank 1 on $M$.

(iii) $d \eta_{1}, d \eta_{2}, d \eta_{3}$ are strictly conformal Killing-Yano tensors of rank 2 on $M$.

(iv) $M$ admits Killing-Yano tensors of rank $(2 k+1)$, for $k \in\{0,1, \ldots, 2 n+1\}$.

Proof. ( $i$ ) From Theorem 3 it follows that $\xi_{1}$ is a unit spacelike and $\xi_{2}, \xi_{3}$ are unit timelike Killing vector fields on $M$.

(ii) It is well-known that a vector field is dual to a conformal Killing-Yano tensor of rank 1 if and only if it is a Killing vector field. Consequently, the statement follows from $(i)$, since $\eta_{1}$, $\eta_{2}, \eta_{3}$ are 1 -forms dual to the vector fields $\xi_{1}, \xi_{2}, \xi_{3}$.

(iii) A direct computation using (3), (4), (5) and (6) leads to:

$$
\nabla_{X} d \eta_{\alpha}=-\frac{1}{4 n+2} X^{*} \wedge d^{*} d \eta_{\alpha}, \quad \alpha \in\{1,2,3\},
$$

for any vector field $X$ on $M$. Therefore, because $d^{2} \eta_{\alpha}=0$, one can write:

$$
\left.\nabla_{X} d \eta_{\alpha}=\frac{1}{3} X\right\lrcorner d\left(d \eta_{\alpha}\right)-\frac{1}{4 n+2} X^{*} \wedge d^{*}\left(d \eta_{\alpha}\right), \quad \alpha \in\{1,2,3\},
$$

and from (2) we deduce that $d \eta_{1}, d \eta_{2}, d \eta_{3}$ are conformal Killing-Yano tensors of rank 2 on $M$. Finally, from Proposition 2 we deduce that they are strictly conformal Killing-Yano tensors on $M$ (i.e. they are not Killing tensor fields); else, if we suppose that $d \eta_{\alpha}$ is a Killing tensor field, then it follows that $\phi_{\alpha}$ is Killing-Yano, which is false.

(iv) Since $\eta_{1}, \eta_{2}, \eta_{3}$ and $d \eta_{1}, d \eta_{2}, d \eta_{3}$ are conformal Killing-Yano tensors on $M$, it follows by direct computations that any linear combination of the forms:

$$
\eta_{1} \wedge\left(d \eta_{1}\right)^{k}, \quad \eta_{2} \wedge\left(d \eta_{2}\right)^{k}, \quad \eta_{3} \wedge\left(d \eta_{3}\right)^{k},
$$

is a Killing-Yano tensor of rank $(2 k+1)$, for all $k \in\{0,1, \ldots, 2 n+1\}$. 
Let us remark that if $M^{4 n+3}$ is a manifold endowed with a mixed 3-Sasakian structure $\left(\left(\phi_{\alpha}, \xi_{\alpha}, \eta_{\alpha}\right)_{\alpha=\overline{1,3}}, g\right)$, then $\xi_{1}+\xi_{2}$ and $\xi_{1}+\xi_{3}$ are lightlike Killing vector fields on $M$. Indeed, since $\left\{\xi_{1}, \xi_{2}, \xi_{3}\right\}$ are orthogonal Killing vector fields, with $\xi_{1}$ unit spacelike vector field and $\xi_{2}, \xi_{3}$ unit timelike vector fields, it is obvious that $\xi_{1}+\xi_{2}$ and $\xi_{1}+\xi_{3}$ are also Killing vector fields and for $\alpha \in\{2,3\}$ one has:

$$
g\left(\xi_{1}+\xi_{\alpha}, \xi_{1}+\xi_{\alpha}\right)=g\left(\xi_{1}, \xi_{1}\right)+2 g\left(\xi_{1}, \xi_{\alpha}\right)+g\left(\xi_{\alpha}, \xi_{\alpha}\right)=1+\epsilon_{\alpha}=0 .
$$

Therefore, $\xi_{1}+\xi_{2}$ and $\xi_{1}+\xi_{3}$ are lightlike Killing vector fields on $M$.

Corollary 2. Let $\left(M^{4 n+3},\left(\phi_{\alpha}, \xi_{\alpha}, \eta_{\alpha}\right)_{\alpha=\overline{1,3}}, g\right)$ be a manifold endowed with a mixed 3-Sasakian structure. Then the distribution spanned by $\left\{\xi_{1}, \xi_{2}, \xi_{3}\right\}$ is integrable and defines a 3 -dimensional Riemannian foliation on $M$, having totally geodesic leaves of constant curvature 1.

Proof. From (9) we obtain:

$$
\left[\xi_{1}, \xi_{2}\right]=0, \quad\left[\xi_{2}, \xi_{3}\right]=2 \xi_{1}, \quad\left[\xi_{3}, \xi_{1}\right]=0,
$$

and consequently, the distribution spanned by $\left\{\xi_{1}, \xi_{2}, \xi_{3}\right\}$ is integrable and defines a 3-dimensional foliation of $M$. On the other hand, since $\xi_{1}, \xi_{2}, \xi_{3}$ are Killing vector fields, it follows that this foliation is Riemannian (see [28]). Moreover, from Theorem 3 we conclude that this foliation has totally geodesic leaves of constant curvature 1.

\section{Some examples}

We give now examples of manifolds endowed with mixed 3-structures and mixed 3-Sasakian structures. In particular, we provide some examples of manifolds which admit Killing-Yano tensors.

Example 1. It is easy to see that if we define $\left(\phi_{\alpha}, \xi_{\alpha}, \eta_{\alpha}\right)_{\alpha=\overline{1,3}}$ in $\mathbb{R}^{3}$ by their matrices:

$$
\begin{aligned}
& \phi_{1}=\left(\begin{array}{ccc}
0 & 0 & 1 \\
0 & 0 & 0 \\
-1 & 0 & 0
\end{array}\right), \quad \phi_{2}=\left(\begin{array}{lll}
0 & 0 & 0 \\
0 & 0 & 1 \\
0 & 1 & 0
\end{array}\right), \quad \phi_{3}=\left(\begin{array}{ccc}
0 & -1 & 0 \\
-1 & 0 & 0 \\
0 & 0 & 0
\end{array}\right), \\
& \xi_{1}=\left(\begin{array}{l}
0 \\
1 \\
0
\end{array}\right), \quad \xi_{2}=\left(\begin{array}{l}
1 \\
0 \\
0
\end{array}\right), \quad \xi_{3}=\left(\begin{array}{l}
0 \\
0 \\
1
\end{array}\right) \text {, } \\
& \eta_{1}=\left(\begin{array}{lll}
0 & 1 & 0
\end{array}\right), \quad \eta_{2}=\left(\begin{array}{lll}
-1 & 0 & 0
\end{array}\right), \quad \eta_{3}=\left(\begin{array}{lll}
0 & 0 & -1
\end{array}\right),
\end{aligned}
$$

then $\left(\phi_{\alpha}, \xi_{\alpha}, \eta_{\alpha}\right)_{\alpha=\overline{1,3}}$ is a mixed 3-structure on $\mathbb{R}^{3}$.

We define now $\left(\phi_{\alpha}^{\prime}, \xi_{\alpha}^{\prime}, \eta_{\alpha}^{\prime}\right)_{\alpha=\overline{1,3}}$ in $\mathbb{R}^{4 n+3}$ by:

$$
\phi_{\alpha}^{\prime}=\left(\begin{array}{cc}
\phi_{\alpha} & 0 \\
0 & J_{\alpha}
\end{array}\right), \quad \xi_{\alpha}^{\prime}=\left(\begin{array}{c}
\xi_{\alpha} \\
0
\end{array}\right), \quad \eta_{\alpha}^{\prime}=\left(\begin{array}{cc}
\eta_{\alpha} & 0
\end{array}\right)
$$

for $\alpha=1,2,3$, where $J_{1}$ is the almost complex structure on $\mathbb{R}^{4 n}$ given by:

$$
J_{1}\left(\left(x_{i}\right)_{i=\overline{1,4 n}}\right)=\left(-x_{2}, x_{1},-x_{4}, x_{3}, \ldots,-x_{4 n-2}, x_{4 n-3},-x_{4 n}, x_{4 n-1}\right),
$$

and $J_{2}, J_{3}$ are almost product structures on $\mathbb{R}^{4 n}$ defined by:

$$
\begin{aligned}
& J_{2}\left(\left(x_{i}\right)_{i=\overline{1,4 n}}\right)=\left(-x_{4 n-1}, x_{4 n},-x_{4 n-3}, x_{4 n-2}, \ldots,-x_{3}, x_{4},-x_{1}, x_{2}\right), \\
& J_{3}\left(\left(x_{i}\right)_{i=\overline{1,4 n}}\right)=\left(x_{4 n}, x_{4 n-1}, x_{4 n-2}, x_{4 n-3}, \ldots, x_{4}, x_{3}, x_{2}, x_{1}\right) .
\end{aligned}
$$

Since $J_{2} J_{1}=-J_{1} J_{2}=J_{3}$, it is easily checked that $\left(\phi_{\alpha}^{\prime}, \xi_{\alpha}^{\prime}, \eta_{\alpha}^{\prime}\right)_{\alpha=\overline{1,3}}$ is a mixed 3 -structure on $\mathbb{R}^{4 n+3}$. 
Example 2. Let $\left(\bar{M}, H=\left(J_{\alpha}\right)_{\alpha=\overline{1,3}}, \bar{g}\right)$ be an almost para-hyperhermitian manifold and $(M, g)$ be a semi-Riemannian hypersurface of $\bar{M}$ with $g=\bar{g}_{\mid M}$, having null co-index.

Then, for any $X \in \Gamma(T M)$ and $\alpha \in\{1,2,3\}$, we have the decomposition:

$$
J_{\alpha} X=\phi_{\alpha} X+F_{\alpha} X
$$

where $\phi_{\alpha} X$ and $F_{\alpha} X$ are the tangent part and the normal part of $J_{\alpha} X$, respectively.

But, since $M$ is a semi-Riemannian hypersurface of $M$ with null co-index, it follows that $T M^{\perp}=\langle N\rangle$, where $N$ is a unit space-like vector field and then $F_{\alpha} X=\eta_{\alpha}(X) N$, where $\eta_{\alpha}(X)=$ $\bar{g}\left(J_{\alpha} X, N\right)$.

Consequently, we have the decomposition:

$$
J_{\alpha} X=\phi_{\alpha} X+\eta_{\alpha}(X) N
$$

We define $\xi_{\alpha}=-J_{\alpha} N$, for all $\alpha \in\{1,2,3\}$ and by straightforward computation it follows that $\left(\left(\phi_{\alpha}, \xi_{\alpha}, \eta_{\alpha}\right)_{\alpha \in\{1,2,3\}}, g\right)$ is a mixed 3-structure on $M$.

We note that in this way we obtain an entire class of examples of manifolds which admit mixed 3-structures, because one can construct semi-Riemannian hypersurfaces of null co-index, both compact and non-compact, by using a standard procedure (see [30]): if $f \in \mathfrak{F}(\bar{M})$ such that $\bar{g}(\operatorname{grad} f, \operatorname{grad} f)>0$ on $M$ and $c$ is a value of $f$, then $M=f^{-1}(c)$ is a semi-Riemannian hypersurface of $\bar{M}$. Moreover, $N=\operatorname{grad} f /|\operatorname{grad} f|$ is a unit spacelike vector field normal to $M$.

We remark that if $M$ is compact then we can provide examples of compact hypersurfaces which can be endowed with mixed 3-structures. In this way we obtain mixed 3-structures on hypersurfaces of the next compact para-hyper-Kähler manifolds of dimension 4: complex tori and primary Kodaira surfaces (see [24]).

Example 3. Let $(\bar{M}, \bar{g})$ be a $(m+2)$-dimensional semi-Riemannian manifold with index $q \in$ $\{1,2, \ldots, m+1\}$ and let $(M, g)$ be a hypersurface of $\bar{M}$, with $g=\bar{g}_{\mid M}$. We say that $M$ is a lightlike hypersurface of $\bar{M}$ if $g$ is of constant rank $m$ (see [1]). Unlike the classical theory of non-degenerate hypersurfaces, in case of lightlike hypersurfaces, the induced metric tensor field $g$ is degenerate.

We consider the vector bundle $T M^{\perp}$ whose fibres are defined by:

$$
T_{p} M^{\perp}=\left\{Y_{p} \in T_{p} \bar{M} \mid \bar{g}_{p}\left(X_{p}, Y_{p}\right)=0, \forall X_{p} \in T_{p} M\right\}, \quad \forall p \in M .
$$

If $S(T M)$ is the complementary distribution of $T M^{\perp}$ in $T M$, which is called the screen distribution, then there exists a unique vector bundle $\operatorname{ltr}(T M)$ of $\operatorname{rank} 1$ over $M$ so that for any non-zero section $\xi$ of $T M^{\perp}$ on a coordinate neighborhood $U \subset M$, there exists a unique section $N$ of $\operatorname{ltr}(T M)$ on $U$ satisfying:

$$
\bar{g}(N, \xi)=1, \quad \bar{g}(N, N)=\bar{g}(W, W)=0, \quad \forall W \in \Gamma\left(S(T M)_{\mid U}\right) .
$$

On another hand, an almost hermitian paraquaternionic manifold is a triple $(\bar{M}, \sigma, \bar{g})$, where $\bar{M}$ is a smooth manifold, $\sigma$ is a rank 3-subbundle of $\operatorname{End}(T \bar{M})$ which is locally spanned by an almost para-hypercomplex structure $H=\left(J_{\alpha}\right)_{\alpha=\overline{1,3}}$ and $\bar{g}$ is a para-hyperhermitian metric with respect to $H$.

In [19] it is proved that there is a mixed 3-structure on any lightlike hypersurface $M$ of an almost hermitian paraquaternionic manifold $(\bar{M}, \sigma, \bar{g})$, such that $\xi$ and $N$ are globally defined on $M$. In particular, the above conditions are satisfied if we consider the lightlike hypersurface $M$ of $\left(R_{4}^{8}, \bar{g},\left\{J_{1}, J_{2}, J_{3}\right\}\right)$ defined by (see [21]):

$$
f\left(\left(t_{i}\right)_{i=\overline{1,7}}\right)=\left(t_{1}, t_{2}, t_{1}+t_{3}, t_{4}+t_{5}, t_{3}+t_{5}+t_{6}, t_{6}+t_{7}, t_{7}, t_{2}\right),
$$


where the structures $J_{1}, J_{2}, J_{3}$ and the metric $\bar{g}$ on $R_{4}^{8}$ are given by:

$$
\begin{aligned}
& \bar{g}\left(\left(x_{i}\right)_{i=\overline{1,8}},\left(y_{i}\right)_{i=\overline{1,8}}\right)=-\sum_{i=1}^{4} x_{i} y_{i}+\sum_{i=5}^{8} x_{i} y_{i}, \\
& J_{1}\left(\left(x_{i}\right)_{i=\overline{1,8}}\right)=\left(-x_{2}, x_{1},-x_{4}, x_{3},-x_{6}, x_{5},-x_{8}, x_{7}\right), \\
& J_{2}\left(\left(x_{i}\right)_{i=\overline{1,8}}\right)=\left(-x_{7}, x_{8},-x_{5}, x_{6},-x_{3}, x_{4},-x_{1}, x_{2}\right), \\
& J_{3}\left(\left(x_{i}\right)_{i=\overline{1,8}}\right)=\left(x_{8}, x_{7}, x_{6}, x_{5}, x_{4}, x_{3}, x_{2}, x_{1}\right) .
\end{aligned}
$$

Example 4. The unit pseudo-sphere $S_{2 n+1}^{4 n+3}$ is the canonical example of manifold with a mixed 3Sasakian structure [22]. This structure is obtained by taking $S_{2 n+1}^{4 n+3}$ as hypersurface of $\left(\mathbb{R}_{2 n+2}^{4 n+4}, \bar{g}\right)$. It is easy to see that on the tangent spaces $T_{p} S_{2 n+1}^{4 n+3}, p \in S_{2 n+1}^{4 n+3}$, the induced metric $g$ is of signature $(2 n+1,2 n+2)$.

If $\left(J_{\alpha}\right)_{\alpha=\overline{1,3}}$ is the canonical para-hypercomplex structure on the $\mathbb{R}_{2 n+2}^{4 n+4}$ given by (13)-(15) and $N$ is the unit spacelike normal vector field to the pseudo-sphere, we can define three vector fields on $S_{2 n+1}^{4 n+3}$ by:

$$
\xi_{\alpha}=-J_{\alpha} N, \quad \alpha=1,2,3 .
$$

If $X$ is a tangent vector to the pseudo-sphere then $J_{\alpha} X$ uniquely decomposes onto the part tangent to the pseudo-sphere and the part parallel to $N$. Denote this decomposition by:

$$
J_{\alpha} X=\phi_{\alpha} X+\eta_{\alpha}(X) N
$$

This defines the 1 -forms $\eta_{\alpha}$ and the tensor fields $\phi_{\alpha}$ on $S_{2 n+1}^{4 n+3}$, where $\alpha=1,2,3$. Now we can easily see that $\left(\phi_{\alpha}, \xi_{\alpha}, \eta_{\alpha}\right)_{\alpha=\overline{1,3}}$ is a mixed 3-Sasakian structure on $S_{2 n+1}^{4 n+3}$.

Example 5. Since we can recognize the unit pseudo-sphere $S_{2 n+1}^{4 n+3}$ as the projective space $P_{2 n+1}^{4 n+3}(\mathbb{R})$, by identifying antipodal points, we also have that $P_{2 n+1}^{4 n+3}(\mathbb{R})$ admits a mixed 3 Sasakian structure.

Applying Corollary 1 we deduce that both $S_{2 n+1}^{4 n+3}$ and $P_{2 n+1}^{4 n+3}(\mathbb{R})$ admit spacelike, timelike and lightlike Killing vector fields, conformal Killing-Yano tensors of rank 1, strictly conformal Killing-Yano tensors of rank 2 and Killing-Yano tensors of rank $(2 k+1)$, for $k \in\{0,1, \ldots, 2 n+1\}$.

\section{Acknowledgements}

We would like to thank the referees for carefully reading the paper and making valuable comments and suggestions. This work was supported by CNCSIS Programs, Romania.

\section{References}

[1] Bejancu A., Duggal K.L., Lightlike submanifolds of semi-Riemannian manifolds and its application, Kluwer, Dortrecht, 1996.

[2] Belgun F., Moroianu A., Semmelmann U., Killing forms on symmetric spaces, Differential Geom. Appl. 24 (2006), 215-222, math.DG/0409104.

[3] Benenti S., Separable dynamical systems: characterization of separability structures on Riemannian manifolds, Rep. Math. Phys. 12 (1977), 311-316.

[4] Blair D.E., Almost contact manifolds with Killing structure tensors, Pacific J. Math. 39 (1971), $285-292$.

[5] Blair D.E., Contact manifolds in Riemannian geometry, Lecture Notes in Mathematics, Vol. 509, SpringerVerlag, Berlin - New York, 1976. 
[6] Boyer C., Galicki K., 3-Sasakian manifolds, in Surveys in Differential Geometry: Essays on Einstein Manifolds, Surv. Differ. Geom., Vol. VI, Int. Press, Boston, MA, 1999, 123-184, hep-th/9810250.

[7] Caldarella A., Pastore A.M., Mixed 3-Sasakian structures and curvature, arXiv:0803.1953.

[8] Cariglia M., Quantum mechanics of Yano tensors: Dirac equation in curved spacetime, Classical Quantum Gravity 21 (2004), 1051-1077, hep-th/0305153.

[9] Carter B., Killing tensor quantum numbers and conserved currents in curved spaces, Phys. Rev. D 16 (1977), 3395-3414.

[10] Carter B., McLenaghan R.G., Generalized total angular momentum operator for Dirac equation in curved space-time, Phys. Rev. D 19 (1979), 1093-1097.

[11] Cortés V., Mayer C., Mohaupt T., Saueressig F., Special geometry of euclidean supersymmetry. II. Hypermultiplets and the c-map, J. High Energy Phys. 2005 (2005), no. 06, 025, 37 pages, hep-th/0503094.

[12] Dunajski M., West S., Anti-self-dual conformal structures in neutral signature, in Recent Developments in Pseudo-Riemannian Geometry, Editors D.V. Alekseevsky and H. Baum, ESI Lect. Math. Phys., Eur. Math. Soc., Zürich, 2008, 113-148, math.DG/0610280.

[13] Floyd R., The dynamics of Kerr fields, PhD Thesis, London University, 1973.

[14] Frolov V.P., Kubiznak D., Higher-dimensional black holes: hidden symmetries and separation of variables, Classical Quantum Gravity 25 (2008), 154005, 22 pages, arXiv:0802.0322.

[15] Gibbons G.W., Rietdijk R.H., van Holten J.W., SUSY in the sky, Nuclear Phys. B 404 (1993), 42-64, hep-th/9303112.

[16] Gray A., Einstein-like manifolds which are not Einstein, Geom. Dedicata 7 (1978), 259-280.

[17] Hull C.M., Actions for (2,1) sigma models and strings, Nuclear Phys. B 509 (1998), 252-272, hep-th/9702067.

[18] Hull C.M., Duality and the signature of space-time, J. High Energy Phys. 1998 (1998), no. 11, 017, 36 pages, hep-th/9807127.

[19] Ianuş S., Mazzocco R., Vîlcu G.E., Real lightlike hypersurfaces of paraquaternionic Kähler manifolds, Mediterr. J. Math. 3 (2006), 581-592.

[20] Ianuş S., Vîlcu G.E., Some constructions of almost para-hyperhermitian structures on manifolds and tangent bundles, Int. J. Geom. Methods Mod. Phys. 5 (2008), 893-903, arXiv:0707.3360.

[21] Ianuş S., Vîlcu G.E., Hypersurfaces of paraquaternionic space forms, J. Gen. Lie Theory Appl. 2 (2008), $175-179$.

[22] Ianuş S., Vîlcu G.E., Paraquaternionic manifolds and mixed 3-structures, in Proceedings of the VIII International Colloquium on Differential Geometry (July 7-21, 2008, Santiago de Compostela), Editors J.A. López and E. García-Río, Analysis and Geometry in Foliated Manifolds, World Scientific, Singapore, 2009, to appear.

[23] Jost J., Riemannian geometry and geometric analysis, 3rd ed., Springer-Verlag, Berlin, 2002.

[24] Kamada H., Neutral hyper-Kähler structures on primary Kodaira surfaces, Tsukuba J. Math. 23 (1999), 321-332.

[25] Kashiwada T., On conformal Killing tensor, Natur. Sci. Rep. Ochanomizu Univ. 19 (1968), 67-74.

[26] Leiva C., Plyushchay M., Nonlinear superconformal symmetry of a fermion in the field of a Dirac monopole, Phys. Lett. B 582 (2004), 135-143, hep-th/0311150.

[27] Matsumoto K., On Lorentzian paracontact manifolds, Bull. Yamagata Univ. Natur. Sci. 12 (1989), $151-156$.

[28] Molino P., Riemannian foliations, Progress in Mathematics, Vol. 73, Birkhäuser Boston, Inc., Boston, MA, 1988.

[29] Moroianu A., Semmelmann U., Twistor forms on Kähler manifolds, Ann. Sc. Norm. Super. Pisa Cl. Sci. (5) 2 (2003), 823-845, math.DG/0204322.

[30] O'Neill B., Semi-Riemannian geometry. With applications to relativity, Pure and Applied Mathematics, Vol. 103, Academic Press, New York, 1983.

[31] Ooguri H., Vafa C., Geometry of $N=2$ strings, Nuclear Phys. B 361 (1991), 469-518.

[32] Penrose R., Naked singularities, Ann. New York Acad. Sci. 224 (1973), 125-134.

[33] Plyushchay M.S., On the nature of fermion-monopole supersymmetry, Phys. Lett. B 485 (2000), 187-192, hep-th/0005122. 
[34] Semmelmann U., Conformal Killing forms on Riemannian manifolds, Math. Z. 245 (2003), 503-527, math.DG/0206117.

[35] Tanimoto M., The role of Killing-Yano tensors in supersymmetric mechanics on a curved manifold, Nuclear Phys. B 442 (1995), 549-560, gr-qc/9501006.

[36] Vaman D., Visinescu M., Supersymmetries and constants of motion in Taub-NUT spinning space, Fortschr. Phys. 47 (1999), 493-514, hep-th/9805116.

[37] Yano K., Some remarks on tensor fields and curvature, Ann. of Math. (2) 55 (1952), 328-347. 